\title{
Rat Diabetic Blood Glucose Level Profile with Stratified Dose Streptozotocin (SD-STZ) and Multi Low Dose Streptozotocin (MLD-STZ) Induction Methods
}

\author{
Nuzulul Hikmah ${ }^{1 *}$, Amandia Dewi Permana Shita ${ }^{1}$, Hafiedz Maulana
}

${ }^{1}$ Biomedical Laboratory, Faculty of Dentistry, Jember University, Indonesia

\begin{abstract}
The prevalence of diabetes increased from year to year. The diabetic animal model was developed to study the pathogenesis and treatment in diabetes. The aim of this study was to determine the profile of diabetic blood glucose level in rat using a stratified dose streptozotocin (STZ-SD) and multi-low dose streptozotocin (MLD-STZ) induction methods. 27 Wistar rats were divided into three groups, the control group, SD-STZ group, and MLD-STZ group. The induction of experimental animal was using the method of SD-STZ and MLD-STZ for five consecutive days intraperitoneally. The SD-STZ method using a stratified dose of 40, 35, 30, 25, and 20 $\mathrm{mg} / \mathrm{Kg}$ of body weight for five days. MLD-STZ methods using a dose of $20 \mathrm{mg} / \mathrm{Kg}$ of body weight for five times. The measurement of blood glucose level (BGL) and body weight (BW) in the animal was made before and on days $2,4,7$, and 14 after STZ induction. The results of this study indicate that the diabetic conditions (BGL $\geq$ $300 \mathrm{mg} / \mathrm{dl}$ ) on the SD-STZ induction method was more stable than MLD-STZ method, and no deaths occurred in experimental animals.
\end{abstract}

Keywords: blood glucose level (BGL), diabetes, streptozotocin (STZ)

\section{INTRODUCTION}

Diabetes is a chronic syndrome characterized by an increased blood glucose level and urine secretion due to insulin deficiency, the effect of insulin action, or both [1]. The number of people with diabetes increases in line with population growth, aging, urbanization, the increasing prevalence of obesity, and physical inactivity [2].

The epidemiology study estimated that by 2030, the prevalence of diabetes mellitus in Indonesia has reached 21.3 million people [2]. This phenomenon has become an important concern for public health, especially to be considered in dentistry treatment.

Streptozotocin (STZ) (is a cytotoxic methyl nitrosourea moiety (N-methyl-N-nitrosourea) which attached to a glucose molecule (2-deoxyglucose). STZ is a diabetogenic agent, used as an experimental of the diabetic animal model. STZ inhibit insulin secretion and cause necrosis of the pancreatic beta cells [3].

STZ induction methods that have been developed at this time are the single dose and multiple low doses

\footnotetext{
*Corresponding author:

Nuzulul Hikmah

Biomedical Laboratory, Faculty of Dentistry, Jember University, Jl. Kalimantan 37 68121, Jember, Indonesia

E-mail: nuzulul.drg@gmail.com
}

STZ with various doses. The single dose method of STZ at a dose of $65 \mathrm{mg} / \mathrm{kg}$ intraperitoneally reported to have limitations, such as the death of rats in each group due to unknown causes [4]. MLD-STZ methods at $40 \mathrm{mg} / \mathrm{kg}$ dose of STZ for five days showed significant diabetic condition. The BGL at week 3 was $334.16 \pm 17.5 \mathrm{mg} / \mathrm{dl}$ and $325.7 \pm 30.8 \mathrm{mg} / \mathrm{dl}$ at week 4 , and no deaths occurred in experimental animals [5]. MLD-STZ methods had been developed using a dose of $20 \mathrm{mg} / \mathrm{kg}$ for five days [6]. The diabetic conditions reported in the second week with BGL average of 549.2 $\pm 11.76 \mathrm{mg} / \mathrm{dl}$ [7].

The failure of obtaining diabetic rats also due to a decline in blood glucose levels in some experimental animals after an increase in blood glucose levels $>300$ $\mathrm{mg} / \mathrm{dl}[4,5]$. A previous study has not been reported whether the induction method used can produce a stable diabetic condition and did not decrease blood glucose levels in animals due to unknown causes, so the animal is not considered diabetic. Therefore, this study will develop an induction method using a stratified dose of STZ (SD-STZ) for 5 consecutive days. The doses are 40, 35, 30, 25, and $20 \mathrm{mg} / \mathrm{kg}$ of body weight intraperitoneally. This method is expected result a more stable condition of hyperglycemia in diabetic conditions. 
The aim of this study was to determine the profile of diabetes blood glucose levels with the induction method stratified dose streptozotocin (STZ-SD) and multi low-dose streptozotocin (MLD-STZ).

\section{MATERIALS AND METHODS}

\section{Experimental Animals and Samples}

Wistar rats (Rattus norvegicus) were used in this study. Inclusion criteria in this study are, (a) healthy rats and no abnormalities, (b) four-month-old-male, (c) weight 250-300 grams. Exclusion criteria are, (a) disability or disorder rats, (b) died during the study, (c) the examination of blood glucose levels on day 3 has not shown a hyperglycemia.

The sample sizes in this study were 27 Wistar rats, which divided into three groups, the control group, SD-STZ treatment group, and MLD-STZ treatment group. All the study procedures were conducted under the guidelines approved by the Ethics Committee of the Dentistry Faculty of Gadjahmada University, Yogyakarta, Indonesia.

\section{Preparation of Streptozotocin (STZ) stock solution}

STZ was dissolved in distilled water and then added to one drop of $0.1 \mathrm{M}$ citrate buffer to obtain STZ solution with a $\mathrm{pH}$ of 4.5 and stored at $4{ }^{\circ} \mathrm{C}$.

\section{Experimental Animals Treatment}

After acclimatized for seven days, the animals were performed at the induction of the MLD-STZ group with a dose of $20 \mathrm{mg} / \mathrm{kg}$ in 5 times for five days. The SD-STZ group was performed at a stratified dose of STZ intraperitoneally. The dose of STZ was started at
$40,35,30,25$, and $20 \mathrm{mg} / \mathrm{kg}$ for five consecutive days. Experimental animals were put in a cage and incubated for 14 days.

\section{Measurement of Blood Glucose Level (BGL) and Body Weight (BW)}

During incubation, the experimental animals were observed blood glucose levels which taken from the tail vein of a rat before and on days 2, 4, 7, and 14 after the induction of STZ. Rats with diabetes are characterized by BGL $\geq 300 \mathrm{mg} / \mathrm{dl}$. The body weight of experimental animals was also measured before and on days $2,4,7$, and 14 after induction of STZ.

\section{Observation}

The BGL was observed using a digital blood glucose level and Glucostick (Gluco-Dr). Experimental animal body weight was measured using a digital scale (Weston).

\section{Statistical Analysis}

Blood glucose levels (BGL) and body weight (BW) of experimental animals was measured before and after treatment. The data were analyzed using the $t$-test with SPSS 20.0 software.

\section{RESULTS AND DISCUSSION Body Weight (BW)}

The measurement of body weight in experimental animals before and after induction of Streptozotocin (STZ) is shown in Figure 1. From Figure 1 it can be seen that the average of body weight in the control group was increased. Different results appear in either

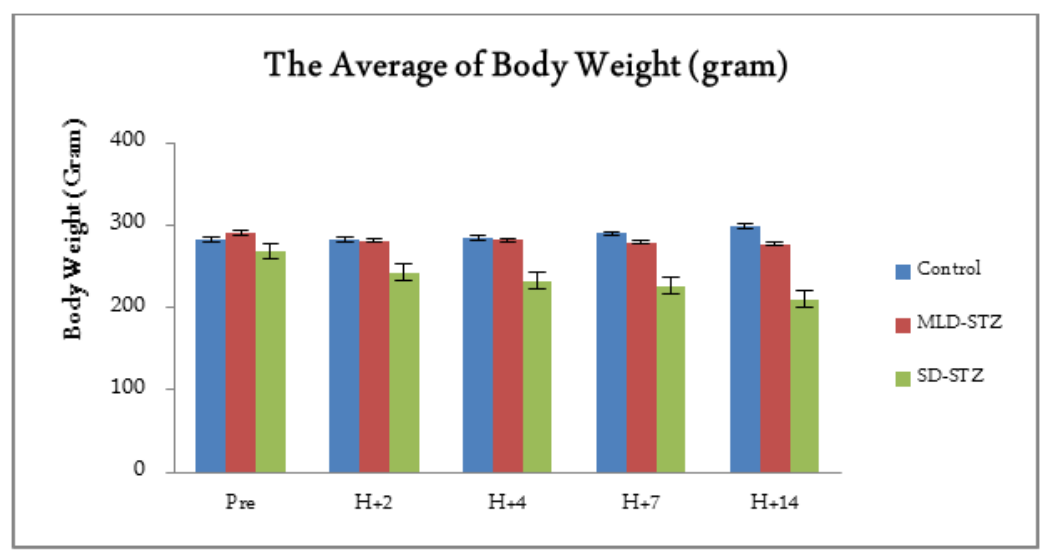

Figure 1. The average of body weight (gram) in the control, MLD-STZ, and SD-STZ groups. Measurements before induction (Pre), measurements after induction at day $2(\mathrm{H}+2), 4(\mathrm{H}+4), 7(\mathrm{H}+7)$, and $14(\mathrm{H}+14)$. The body weight of SD-STZ group experienced a sharp decline compared with the MLD-STZ group. 


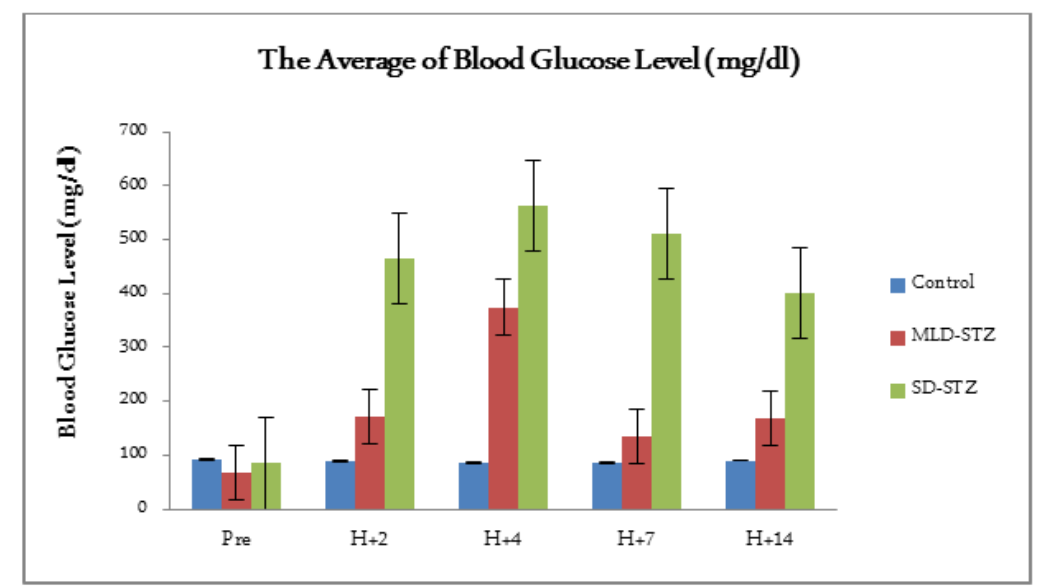

Figure 2. The average of blood glucose level $(\mathrm{mg} / \mathrm{dl})$ in the control, MLD-STZ, and SD-STZ groups. The blood glucose level in the SD-STZ group was more stable than MLD-STZ group above $300 \mathrm{mg} / \mathrm{dl}$, both on days 2, 4, 7 and 14.

MLD-STZ or SD-STZ group. The SD-STZ group experienced a sharp decline compared with the MLD-STZ group.

\section{Blood Glucose Levels (BGL)}

The measurement of blood glucose level in experimental animals before and after the induction of STZ was shown in Figure 2. Figure 2 showed the average of blood glucose levels in the control group was in normal conditions. There was an increased of the MLDSTZ group blood glucose levels at day four after induction $(374.2 \mathrm{mg} / \mathrm{dl})$. The BGL at day 14 after induction was decreased back $(169.1 \mathrm{mg} / \mathrm{dl})$.

In the SD-STZ group, there were an increased of blood glucose levels on day $2(464.7 \mathrm{mg} / \mathrm{dl})$ and day 14 $(563 \mathrm{mg} / \mathrm{dl})$. The average of blood glucose levels in SD-STZ group at day 7 and 14 were decreased by 511.9 $\mathrm{mg} / \mathrm{dl}$ and $401 \mathrm{mg} / \mathrm{dl}$, but still in the category of diabetic rats. These results indicate that the BGL in the SD-STZ group was more stable than MLD-STZ group above $300 \mathrm{mg} / \mathrm{dl}$, both on days 2, 4, 7 and 14 .

Statistical analysis showed a significant difference of BGL before and after induction in the SD-STZ and MLD-STZ group $(\mathrm{p}<0.05)$. Statistical analysis also showed no significant differences in the SD-STZ group between $\mathrm{H}+4-\mathrm{H}+14$ and $\mathrm{H}+7-\mathrm{H}+14$ ( $>>0.05)$.

This study used a diabetic rat model of 2, 4, 7 and 14 days. The difference of time was to determine the differences in blood glucose levels because of a short period of diabetes. The aged conversion of rat model with human age showed that 20-day old rats are approximately equal to two years of human life [8]. Therefore, in 14 days diabetic rat model was analogous to a human who have diabetes for more than 17 months.

The results of this study showed that the average of body weight in the control group was increased. Different results appear in both MLD-STZ and SD-STZ groups. The SD-STZ group experienced a sharp decline compared with MLD-STZ group (Figure 1). The results are consistent with previous studies that the STZ induced diabetic rats shown a significant decrease in body weight at week 2 after STZ induction [9]. The loss of body weight because of induction of STZ was associated with an increase in muscle wasting (gluconeogenesis and glycogenolysis) and the loss of tissue proteins $[10,11]$. Insulin deficiency can cause a variety of metabolic disorders, including the increased blood glucose level, decreased in protein content and increased of cholesterol as well as triglyceride levels [12].

The results of this study showed that the average of blood glucose levels in SD-STZ group was more stable above $300 \mathrm{mg} / \mathrm{dl}$ compared with the MLD-STZ group (Figure 2). These results indicated that the SD-STZ method produces hyperglycemia conditions (BGL $\geq$ $300 \mathrm{mg} / \mathrm{dl}$ ) were more stable than using the MLD-STZ methods. Statistical analysis showed a significant difference of BGL before and after induction, both in SDSTZ and MLD-STZ group. Statistical analysis results indicated that no significant differences in the blood glucose levels on days 2, 4, 7, and 14 with SD-STZ and MLD-STZ induction methods.

Blood glucose levels were used to diagnose diabetes. Hyperglycemia in diabetes was caused by insulin secretion deficiency by pancreatic beta cell dysfunction or insulin act resistance in the liver and muscle, or combination of both [13]. 
Diabetic condition on SD-STZ has shown a more stable hyperglycemia condition. This was probably caused by a greater dose of STZ. However, it gave rise toward smaller doses. The stratified dose was given as an adaptation response in a diabetic rat model of STZ administration. The Less stable hyperglycemia condition in MLD-STZ induction method was possibly because of repeated administration of smaller doses, resulting in a decrease in blood glucose levels at days 7 and 14 after induction. A blood glucose level condition which less stable and reversible was possible happened because of the regeneration process of pancreatic beta cell damage. Some of the material that caused diabetes in experimental animals such as alloxan can cause a spontaneous regeneration of pancreatic beta cells [14].

Streptozotocin (STZ) is a diabetogenic agent, which is used as an experimental diabetes mellitus in laboratory animals. STZ inhibit insulin secretion and cause necrosis of the pancreatic beta cells [3]. STZ inhibit the biosynthesis and secretion of insulin through interference with the glucose metabolism and oxygen consumption [3]. Streptozotocin-induced diabetes mellitus (STZ-DM) which is caused by the pancreatic beta cell destruction was similar to type 1 of diabetes in humans. It was characterized by hyperglycemia, glycosuria, polyphagia, hypoinsulinemia, hyperlipidemia, and body weight loss. STZ-DM also shows some complications that include increased susceptibility to infection, cardiovascular disease, retinopathy, changes in angiogenesis, wound healing disorders, decreased expression of growth factors, and decreased bone formation $[15,16]$.

The advantages of STZ induction on diabetic conditions were more stable than other materials. A diabetic rat model of STZ induction can be used as an experimental study for the long term. The disadvantages of diabetic STZ induction are the elevation of mortality rate, and the failure to get diabetic conditions [14].

\section{CONCLUSIONS}

From this study, it can be concluded that the rat diabetic conditions (BGL $\geq 300 \mathrm{mg} / \mathrm{dl}$ ) of SD-STZ induction method were more stable than MLD-STZ method. There were no deaths occurred in experimental animals.

\section{ACKNOWLEDGMENT}

We would like to send our gratitude to DR. drg. Nur Permatasari, MS., and DR. Dra. Sri Widyarti, MSi., for their valuable help in conducting this study.

\section{REFERENCES}

1. Halliwel B and Gutteridge JMC (1999) Free radical in biology and medicine. 3rd Edition. New York: Oxford University Press. p. 639-45.

2. Wild S, Roglic G, Green A, Sicree R, and King H (2004) Global prevalence of diabetes estimates for the year 2000 and projections for 2030. Diabetes Care 27: 1047-1053.

3. Lenzen S (2007) Alloxan and streptozotocin diabetes. [http://www.saw-leipzig.de/endo_07-lenzen_3.pdf].

4. Nurdiana, Permatasari N, Setyawati, Ali M (1998) Streptozotocin effect as diabetogenik agent on wistar rats with intraperitoneal and intravena inductions. Majalah Kedokteran Unibraw. 14(2): 66-73.

5. Arora S, Ojha K, Vohora D (2009) Characterisation of streptozotocin induced diabetes mellitus in swiss albino mice. Global Journal of Pharmacology. 3(2): 81-84.

6. Aulanni'am (2011) Superoxide dismutase (SOD) activity and histological pancreas of type 1 diabetes mellitus rats which get the temu giring (Curcuma heyneana) extract treatment. Media Kedokteran Hewan. 27(1).

7. Herawati A, Aulanni'am, and Prasetyawan S (2012) The role of $\mathrm{d}$-alpha tocopherol therapy to blood glucose levels and levels of MDA (malondialdehyde) in diabetes mellitus type 1 rats by MLD-STZ. Veterinaria Medica. 5(3).

8. Rajashree R, Kholkute SD, Goudar SS (2011) Effects duration of diabetes on behavioural and cognitive parameters in streptozotocin-induced juvenile diabetic rats. Malaysian J Med Sci. 18(4): 26-31.

9. Minaiyan M, Ghannadi A, Movahedian A, Hakim-Elahi I (2014) Effect of Hordeum vulgare L. (Barley) on blood glucose levels of normal and STZ-induced diabetic rats. Research in Pharmaceutical Sciences 9(3): 173-178.

10. Kato A, Minoshima Y, Yamamoto J, Adachi I, Watson A, Nash R (2008) Protective effects of dietary chamomile tea on diabetic complications. J. Agric. Food Chemistry. 56: 8206-8211.

11. Emam MA (2012) Comparative evaluation of antidiabetic activity of Rosmarinus officinalis L. and Chamomile recutita in streptozotocin induced diabetic rats. Agric.Biol.J.N.Am. 3(6): 247-252.

12. Tenpe CR and Yeole PG (2009) Comparative evaluation of antidiabetic activity of some marketed polyherbal formulations in alloxan induced diabetic rats. International Journal of Pharm Tech Research. 1(1): 43-49.

13. Mealey BL and Ocampo GL (2007) Diabetes mellitus and periodontal disease. Periodontology 2000, 44: 127-153.

14. Kumar S, Singh R, Vasudeva N, and Sharma S (2012) Acute and chronic animal models for the evaluation of anti-diabetic agents. Cardiovascular Diabetology. 11: 9.

15. Kunjathoor VV, Wilson DL, and LeBoeuf RC (1996) Increased atherosclerosis in streptozotocin-induced diabetic 
mice. J. Clin. Invest. 97(7): 1767-1773.

16. Lu H, Kraut D, Gerstenfeld LC, and Graves DT (2003) Diabetes interferes with the bone formation by affecting the expression of transcription factors that regulate osteoblast differentiation. Endocrinology. 144(1): 346-352. 